\title{
The adhesion receptor CD44 promotes atherosclerosis by mediating inflammatory cell recruitment and vascular cell activation
}

\author{
Carolyn A. Cuff, ${ }^{1}$ Devashish Kothapalli, ${ }^{2}$ Ijeoma Azonobi, ${ }^{1}$ Sam Chun, ${ }^{1}$ \\ Yuanming Zhang, ${ }^{3}$ Richard Belkin, ${ }^{4}$ Christine Yeh, ${ }^{1}$ Anthony Secreto, ${ }^{4}$ \\ Richard K. Assoian, ${ }^{2}$ Daniel J. Rader, ${ }^{4}$ and Ellen Puré ${ }^{1,5}$ \\ ${ }^{1}$ The Wistar Institute, \\ ${ }^{2}$ Department of Pharmacology, \\ ${ }^{3}$ Cell and Molecular Biology Graduate Group, and \\ ${ }^{4}$ Department of Medicine, University of Pennsylvania School of Medicine, Philadelphia, Pennsylvania, USA \\ ${ }^{5}$ The Ludwig Institute for Cancer Research, New York, New York, USA
}

Address correspondence to: Ellen Puré, The Wistar Institute, Room 368, 3601 Spruce Street, Philadelphia, Pennsylvania 19104, USA. Phone: (215) 898-1570; Fax: (215) 898-3937; E-mail: pure@mail.wistar.upenn.edu.

Carolyn A. Cuff's present address is: Bristol-Meyers Squibb Pharmaceutical Research Institute, Department of Vascular Biology, Hopewell, New Jersey, USA.

Received for publication February 2, 2001, and accepted in revised form August 13, 2001.

\begin{abstract}
Atherosclerosis causes most acute coronary syndromes and strokes. The pathogenesis of atherosclerosis includes recruitment of inflammatory cells to the vessel wall and activation of vascular cells. CD44 is an adhesion protein expressed on inflammatory and vascular cells. CD44 supports the adhesion of activated lymphocytes to endothelium and smooth muscle cells. Furthermore, ligation of CD44 induces activation of both inflammatory and vascular cells. To assess the potential contribution of CD44 to atherosclerosis, we bred CD44-null mice to atherosclerosis-prone apoE-deficient mice. We found a 50-70\% reduction in aortic lesions in CD44-null mice compared with CD44 heterozygote and wild-type littermates. We demonstrate that CD44 promotes the recruitment of macrophages to atherosclerotic lesions. Furthermore, we show that CD44 is required for phenotypic dedifferentiation of medial smooth muscle cells to the "synthetic" state as measured by expression of VCAM-1. Finally, we demonstrate that hyaluronan, the principal ligand for CD44, is upregulated in atherosclerotic lesions of apoE-deficient mice and that the low-molecular-weight proinflammatory forms of hyaluronan stimulate VCAM-1 expression and proliferation of cultured primary aortic smooth muscle cells, whereas high-molecular-weight forms of hyaluronan inhibit smooth muscle cell proliferation. We conclude that $\mathrm{CD} 44$ plays a critical role in the progression of atherosclerosis through multiple mechanisms.
\end{abstract}

J. Clin. Invest. 108:1031-1040 (2001). DOI:10.1172/JCI200112455.

\section{Introduction}

Multiple risk factors for development of atherosclerosis have been identified, including elevated levels of LDL cholesterol. Although lipid-lowering drugs have had a major impact by decreasing coronary events by about $30 \%$ over 5 years (1), it will be necessary to better define the molecular mechanisms of atherogenesis to provide a rational basis for design of novel therapies to further reduce risk. Atherosclerosis is also characterized by the focal recruitment of macrophages and $T$ lymphocytes that secrete soluble mediators that perpetuate the inflammatory response underlying the progression of atherosclerotic lesions (2). Vascular cells also play a critical part in disease progression. Activated endothelial cells express adhesion molecules required for the recruitment of inflammatory cells to the vessel wall. Normally vascular smooth muscle cells (SMCs) reside in the media in a quiescent or contractile state. Atherosclerotic lesions are characterized by dedifferentiation of SMCs to a synthetic state characterized by proliferation and migration into the neointima (3). In addition, SMC dedifferentiation is associated with downregulation of $\alpha$-smooth muscle actin and upregulation of adhesion molecules (4), as well as matrix components such as laminin (3).

CD44 is expressed on both inflammatory and vascular cells and can mediate adhesion of $\mathrm{T}$ lymphocytes to endothelium $(5,6)$ and SMCs $(7)$, release of inflammatory mediators from macrophages (8-10) and $\mathrm{T}$ lymphocytes (11-13), and proliferation of vascular SMCs (14). The principal ligand of CD44 is hyaluronan (HA), a major glycosaminoglycan constituent of extracellular matrix that is upregulated within atherosclerotic lesions (14-16).

The previous evidence that $\mathrm{CD} 44$ can potentially mediate several atherogenic processes including inflammatory cell recruitment and cellular activation $(5,8,9)$, together with the finding that the principal lig- 
and of CD44, HA, is upregulated in atherosclerotic lesions (14-16), led us to hypothesize that CD44 may promote atherogenesis. We report that $C D 44$-null mice had markedly reduced atherosclerosis compared with CD44 heterozygote and wild-type littermates. Furthermore, we demonstrate that CD44 is critical for the recruitment of macrophages to atherosclerotic lesions and plays a role in the dedifferentiation of SMCs to the synthetic phenotype. Furthermore, HA regulates SMC proliferation and VCAM-1 expression in vitro. We conclude that CD44 plays an important role in the pathogenesis of atherosclerosis.

\section{Methods}

Animals. CD44-deficient mice (17), sixth-generation backcross to C57BL/6, were bred to apoE-deficient mice (tenth-generation C57BL/6; The Jackson Laboratory, Bar Harbor, Maine, USA) to generate third-generation mice (seventh-generation backcross to C57BL/6) that were all $\mathrm{apoE}^{-/-}$and either wild-type, or heterozygous, or homozygous null at the $C D 44$ locus. All comparisons of atherosclerosis were made with littermate controls. Mice were fed a normal chow diet and atherosclerosis was analyzed at 20 or 24 weeks of age as indicated. C57BL/6 mice were purchased from Taconic Farms (Germantown, New York, USA). Animals were euthanized by inhalation of $\mathrm{CO}_{2}$, gently perfused with PBS, and the aorta and heart were dissected. The aorta was fixed in $10 \%$ formalin and the hearts were embedded in OCT and frozen. Mice were housed in the Wistar Institute animal facility in a specific pathogen-free environment. All procedures were approved by the Institute Animal Care and Use Committee.

Analysis of atherosclerosis. The extent of atherosclerosis in the thoracic and abdominal aorta was measured en face using methods described previously (18). Cholesterol deposits within the aorta were visualized by staining with Sudan IV, and the extent of lesion was expressed as percentage of aorta covered by lesion. Atherosclerotic lesion area in the aortic root was measured by the aortic root assay (18) in which $8-\mu \mathrm{m}$ sections of the aortic root were collected on 12-well masked slides (Carlson Scientific Inc., Peotone, Illinois, USA) and immunostained with Ab's described below to visualize the atherosclerotic lesions. A minimum of four sections spaced over approximately $300 \mu \mathrm{m}$ of the proximal aorta were analyzed for each animal by a blinded observer and the average lesion area within each animal was determined by computer-assisted morphometric analysis (Phase 3 Imaging software; Phase 3 Imaging Systems, Glen Mills, Pennsylvania, USA).

Cholesterol analysis. Serum total cholesterol concentrations were determined using enzymatic reagents (Sigma Chemical Co., St. Louis, Missouri, USA) on a Cobas Fara II autoanalyzer (Roche Diagnostic Systems Inc., Nutley, New Jersey, USA). Lipoprotein cholesterol distributions were evaluated in pooled serum samples $(120 \mu \mathrm{l})$ from four mice in each group after fractionation by fast protein liquid chromatography gel filtration (Amersham
Pharmacia Biotech AB, Uppsala, Sweden) on two Superose 6 columns connected in series (19). Fractions were collected and cholesterol concentrations were determined with an enzymatic-based assay kit (Wako Chemicals USA, Richmond, Virginia, USA).

SMC cultures. Vascular SMCs were isolated from the aorta of C57BL/6 or CD44-deficient mice by explant culture by removing the adventitial layer, longitudinally dissecting the aorta, and placing 2- to $6-\mathrm{mm}$ pieces face down under coverslips in 40\% DMEM, 40\% F12, 20\% heat-inactivated FCS, $25 \mathrm{mM}$ HEPES, penicillin, and streptomycin. Fresh media (15\% serum) was applied every 3-4 days, and cells were passaged at $80 \%$ confluence by trypsin/EDTA treatment. Experiments were performed on cells from passage two or three. The purity of each population was assessed by expression of $\alpha$-smooth muscle actin as detected by flow cytometry, and cultures that were greater than $95 \%$ positive were used for experiments. For determination of phenotype, SMCs were serum deprived by culturing in $0.2 \%$ serum for 2 days before stimulation with low-molecularweight HA (LMW-HA; ICN Biomedicals Aurora, Ohio, USA), high-molecular-weight HA (HMW-HA; Pharmacia \& Upjohn, Peapack, New Jersey, USA), or TNF- $\alpha$ $(100 \mathrm{U} / \mathrm{ml}$; R\&D Systems Inc., Minneapolis, Minnesota, USA). All treatments were carried out in the presence of polymixin B (Sigma Chemical Co.) to eliminate the effects of potential LPS contamination, which was monitored by the Limulus endotoxin assay. VCAM-1 levels were analyzed by flow cytometry. NF- $\mathrm{KB}$ activation was analyzed by electromobility-shift assay as described previously (20) using a ${ }^{32}$ P-labeled oligonucleotide representing the consensus sequence of the NF-KB-binding site (Santa Cruz Biotechnology Inc., Santa Cruz, California, USA) as a probe.

For measurement of proliferation, SMCs were made quiescent by starving them in serum-free media containing $0.1 \%$ BSA for 48 hours. Quiescent SMCs were seeded on coverslips in media containing either $1 \%$ FCS or 10\% FCS and treated with LMW-HA or HMW-HA for 48 hours. The rate of S-phase entry was determined using standard immunofluorescence procedures (21) with anti-5-bromo-2'-deoxyuridine (anti-BrdU) Ab. In some experiments, a blocking anti-CD44 Ab, KM81, or an irrelevant isotype-matched $\mathrm{Ab}(20 \mu \mathrm{g} / \mathrm{ml})$, were added for the duration of treatment with HA.

Macrophage recruitment assay. Macrophage migration to atherosclerotic lesions was determined as described previously (22). Briefly, thioglycollate-elicited peritoneal macrophages were adherence purified and labeled with either ${ }^{111}$ indium-oxyquinolone (Nycomed Amersham, Princeton, New Jersey, USA) according to a method described previously (23) or $2 \mu \mathrm{m}$ yellow-green fluorescent beads according to the manufacturer's recommendation (Molecular Probes Inc., Eugene, Oregon, USA). Cells $\left(2 \times 10^{6}\right)$ were injected intravenously via the orbital plexus. Radioactivity in the aortic arch, spleen, and liver was quantified with a gamma-counter (Hewlett-Packard (Palo Alto, California, USA). Data was normalized for 

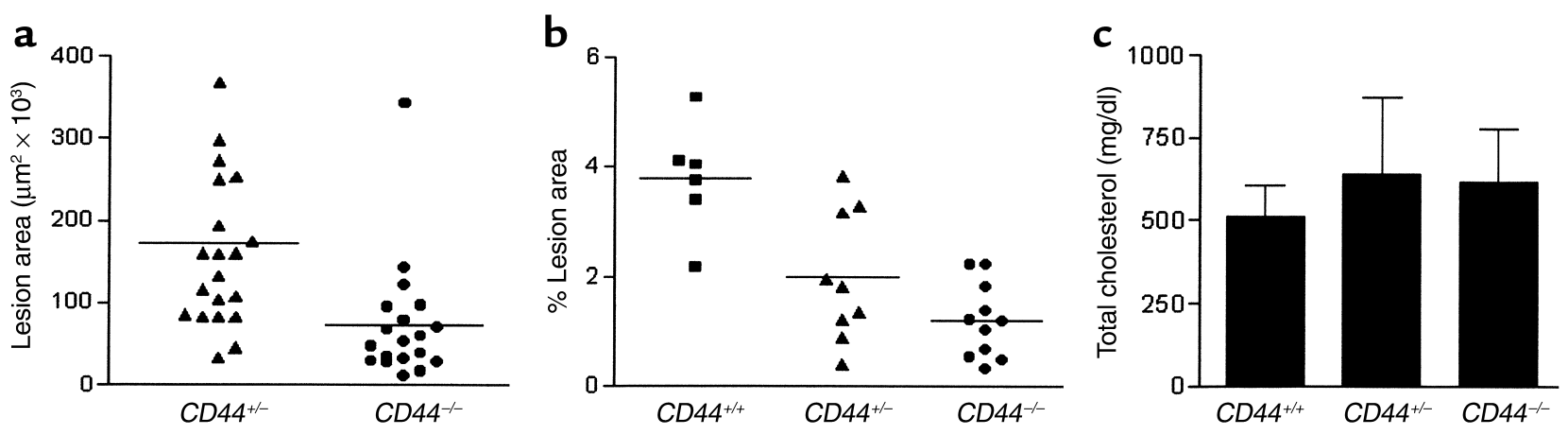

Figure 1

Genetic deficiency in CD44 results in marked reduction of atherosclerosis in apoE-deficient mice. (a) Atherosclerotic lesion area in CD44 ${ }^{+}-$ $(n=21)$ and $C D 44^{-/-}(n=19)$ mice was quantitated by aortic root assay; $P=<0.01$. (b) Atherosclerotic lesion area in the entire thoracicabdominal aorta of $\mathrm{CD} 44^{+/+}(n=6), C D 44^{+/-}(n=9)$, and $C D 44^{-/-}(n=10)$ mice quantitated by en face analysis. (c) Total cholesterol levels in the plasma of $C D 44^{+/+}(n=18), C D 44^{+/-}(n=20)$, and $C D 44^{-/-}(n=19)$ mice were equivalent.

radioactivity detected in the spleen and liver to account for variations in injections. Alternatively, hematopoietic cells were isolated from bone marrow and labeled with the fluorochrome calcein (Molecular Probes Inc.). In experiments in which cells were fluorescently labeled, the aortic arch was frozen in OCT and cryosections through the entire aortic arch were analyzed by immunofluorescence microscopy for quantitation and localization of macrophages within lesions. Injections were normalized by the amount of fluorescence present in liver and spleen homogenates as detected by fluorimetry.

Immunobistochemistry. Eight-micrometer tissue sections were fixed in acetone, air dried, and treated with $0.3 \% \mathrm{H}_{2} \mathrm{O}_{2}$ for 20 minutes. Tissues were rehydrated with PBS and blocked with 3\% BSA in PBS for 30 minutes. Following 1-hour incubation with primary Ab's, sections were incubated with biotinylated secondary Ab's (Vector Laboratories Inc., Burlingame, California, USA). Horseradish-peroxidase-conjugated (HRP-conjugated) ABC amplification system (Vector Laboratories Inc.) was used with all Ab's, and reactivity was visualized by 3,3-diaminobenzidine (DAB) substrate (Polysciences Inc., Warrington, Pennsylvania, USA).

Immunobistochemical reagents. Ab's used were as follows: FITC-conjugated anti- $\alpha$-smooth muscle actin (10 $\mu \mathrm{g} / \mathrm{ml}$; Sigma Chemical Co.); biotinylated goat anti-FITC $(2.5 \mu \mathrm{g} / \mathrm{ml}$; Sigma Chemical Co. $)$ CD44, IM7 $(20 \mu \mathrm{g} / \mathrm{ml}$; American Type Tissue Collection [ATTC], Manassas, Virginia, USA), and CD44, KM81 $(20 \mu \mathrm{g} / \mathrm{ml}$; ATCC) VCAM-1, $429(20 \mu \mathrm{g} / \mathrm{ml}$, PharMingen, San Diego, California, USA); ICAM-1, YN1 $(20 \mu \mathrm{g} / \mathrm{ml}$; ATCC); PECAM-1 (20 $\mu \mathrm{g} / \mathrm{ml}$; ATTC); laminin (0.4 $\mu \mathrm{g} / \mathrm{ml}$; Sigma Chemical Co.); collagen type I and IV (0.5 $\mu \mathrm{g} / \mathrm{ml}$;; Sigma Chemical Co.); CD18, 2E6 (20 $\mu \mathrm{g} / \mathrm{ml}$; ATCC); CD11c, N418 (20 $\mu \mathrm{g} / \mathrm{ml}$; ATCC); Mac-1 (20 $\mu \mathrm{g} / \mathrm{ml}$; ATCC), anti-BrdU (Amersham Pharmacia Biotech, Piscataway, New Jersey, USA); and FITC-conjugated anti-mouse IgG (Jackson ImmunoResearch Laboratories Inc., West Grove, Pennsylvania, USA).

HA levels were detected with cartilage-derived HA-binding protein (HA-BP) (24) $(5 \mu \mathrm{g} / \mathrm{ml}$; Seigagaku, Tokyo,
Japan) followed by HRP-conjugated avidin (Zymed Laboratories Inc., South San Francisco, California, USA).

Statistics. All data were analyzed by Student's $t$ test. A $P$ value less than 0.05 was considered statistically significant.

\section{Results}

Genetic deficiency in CD44 results in a marked reduction of atherosclerosis in apoE-deficient mice. We bred mice with a null mutation in CD44 (17) to apoE-deficient mice and analyzed the extent of atherosclerotic lesions. Strikingly, at 20 weeks of age in mice maintained on a normal chow diet, lesion area in the aortic root of CD44null mice was reduced $70 \%$ compared with $C D 44$ heterozygote littermates $(P<0.01$; Figure 1a). This reduction in atherosclerotic lesions was confirmed and extended by en face analysis of the thoracic abdominal aorta. In this assay, lesion area was reduced in CD44null mice by $49 \%$ compared with heterozygote littermates $(P<0.05)$ and $75 \%$ compared with wild-type littermates $(P<0.01$; see Figure $1 \mathrm{~b})$, indicating that CD44 regulated atherosclerotic lesion formation in a gene dosage-dependent manner. Atherosclerotic lesions in the CD44-null and CD44 heterozygote mice then progressed at similar rates from 20 to 24 weeks ( 2.5 -fold increase). At 24 weeks, lesions from CD44-deficient mice were still reduced by $37 \%$ compared with $C D 44$ heterozygote littermates $(P<0.05, n=10$; data not shown). Deficiency at the CD44 locus did not affect lipid metabolism because analysis of plasma cholesterol levels indicated that there was no difference in either the total plasma cholesterol levels nor was their a difference in the profile of lipoproteins (Figure 1c). Together these data indicate that CD44 promotes atherogenesis and that the reduction in atherosclerotic lesions observed in CD44-null mice is not due to alterations in plasma lipid metabolism.

To determine if CD44 plays a role in regulating the morphology of atherosclerotic lesions we performed immunohistochemical analysis of the aortic root. The reduction in lesion area in the CD44-null mice was clearly apparent in this analysis as well (Figure 2). How- 

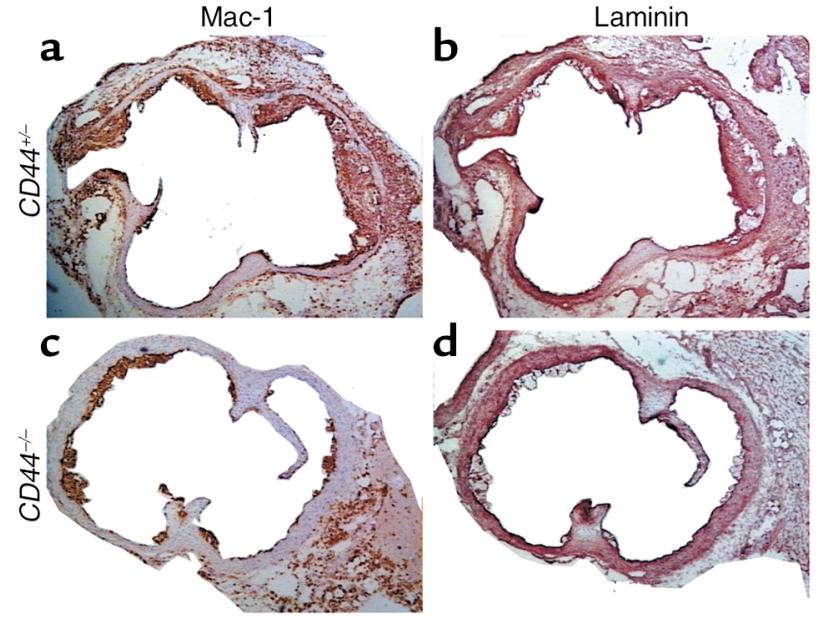

\section{Figure 2}

Morphology of atherosclerotic lesions was not affected in CD44-deficient mice. Aortic root sections from $\mathrm{CD}_{44^{+/-}}(\mathbf{a}$ and $\mathbf{b})$ and $\mathrm{CD} 44^{-/-}$ (c and $\mathbf{d}$ ) mice were immunostained with the macrophage marker Mac-1 ( $\mathbf{a}$ and $\mathbf{c}$ ) to visualize macrophage-rich areas as well as the matrix component laminin (b and d) to identify fibrotic areas. Representative of $21 \mathrm{CD}_{4} 4^{+/-}$and $19 \mathrm{CD} 44^{-/-}$mice. $\times 50$.

ever, there were no obvious differences in the relative composition of the lesions. Thus, lesions in CD44-null and $C D 44$ heterozygote mice contained a comparable proportion of macrophage-rich areas identified with Mac-1 (Figure 2, a and c) and CD11c-specific Ab's that identify foam cells (25) (data not shown) and a comparable proportion of matrix-rich fibrotic areas detected with laminin (Figure 2, b and d) and collagen-specific $\mathrm{Ab}$ 's (data not shown).

HA and CD44 regulate SMC VCAM-1 expression in atherosclerotic lesions. VCAM-1 expression was previously implicated as a marker of transition of SMCs to the synthetic phenotype within atherosclerotic lesions in humans and rabbits (3). We found that VCAM-1, but not ICAM-1, was selectively upregulated on SMCs in association with atherosclerotic lesions, but not expressed on medial smooth muscle in adjacent areas of the vessel devoid of lesion. VCAM-1 was initially detected on the ablumenal medial SMCs prior to or coincident with the earliest detectable recruitment of leukocytes (Figure 3, a and b). Expression of VCAM-1 extended to the full thickness of the media as lesions progressed (Figure 3c) but remained restricted to SMC underlying lesions. The SMCs in the neointima of lesions also expressed VCAM-1. Thus, medial smooth muscle VCAM-1 expression identifies early lesions and may be an early phenotypic manifestation of SMC dedifferentiation that is maintained on SMC in advanced lesions (26). CD44 was expressed by smooth muscle and endothelial cells in normal vessels and upregulated on SMCs underlying lesions and on macrophages within lesions (Figure 3f), as determined by immunoreactivity with cell-specific markers in serial sections.

Analysis of serial sections demonstrated an accumulation of HA that colocalized with VCAM-1 in athero- sclerotic lesions (Figure 4, a-f). These results raised the possibility that $\mathrm{CD} 44$, the principal HA receptor, might regulate the expression VCAM-1. Indeed, we found that the upregulation of VCAM- 1 by SMCs in lesions from CD44-deficient mice was dramatically attenuated compared with the marked upregulation of VCAM-1 on SMCs in lesions from CD44 heterozygote mice (Figure 5). This differential regulation of VCAM-1 was evident in comparing intermediate and advanced lesions (Figure 5 , a and b vs. $\mathrm{d}$ and e) as well as early foam cell lesions (Figure 5, c vs. f) from heterozygous and CD44null mice. Thus, marked upregulation of VCAM-1 on smooth muscle cells was evident in all lesions and at all stages in the many wild-type (Figure 3) and nine heterozygous (Figure $5, \mathrm{a}-\mathrm{c}$ ) mice that have been analyzed for VCAM-1 expression. In contrast, marked upregulation of VCAM-1 was not detected in any lesions at any stage in the aortas isolated from the seven CD44-null mice analyzed. Furthermore, the differential regulation of VCAM-1 was evident in comparing lesions of comparable size and complexity at the various stages despite the fact that the lesions from the CD44-null mice and the CD44 heterozygote mice exhibited comparable morphologies typical of intermediate to

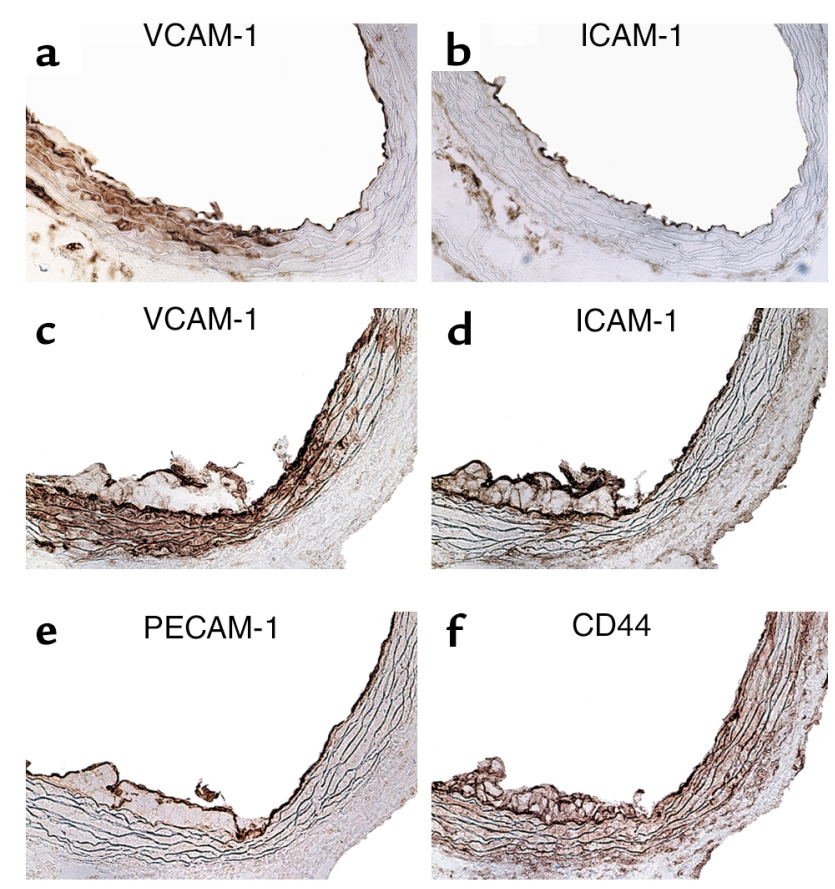

\section{Figure 3}

Upregulation of VCAM-1 on vascular SMCs is an early and persistent marker of atherosclerosis. Immunohistochemistry of the arch region of aorta isolated from 6-week (a and b) or 16-week (c-f) apoE-deficient mice with anti-VCAM-1 (a and c), anti-ICAM-1 (b and d), anti-PECAM-1 (e), and anti-CD44 (f) Ab's. VCAM-1 is selectively and markedly upregulated on the vascular SMCs in atherosclerosis-prone areas of the aorta, including on medial SMCs underlying established lesions, while ICAM-1 expression is restricted to endothelial cells. CD44 was expressed on macrophage-derived foam cells and upregulated on vascular SMCs underlying lesion. Representative sections from five to seven mice. $\times 100$. 

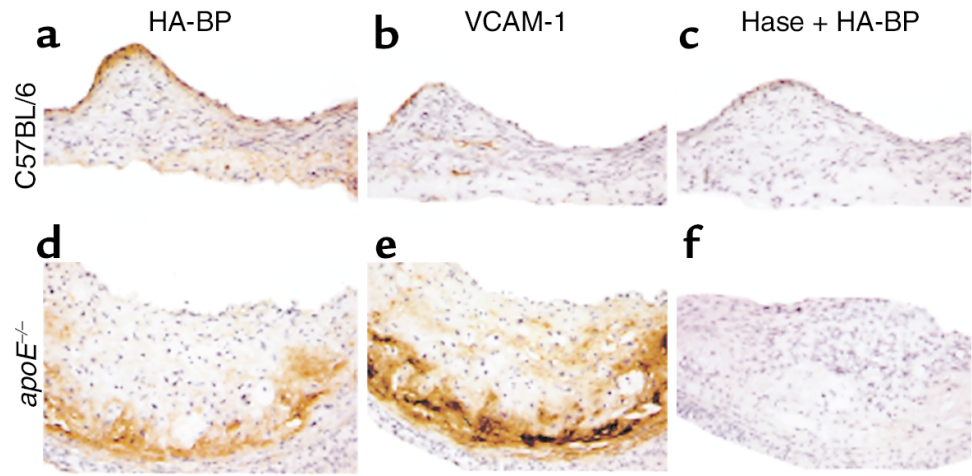

f

\section{Figure 4}

VCAM-1 is upregulated within HA-rich regions of atherosclerotic lesions in apoEdeficient mice. Histochemical detection of HA and VCAM- 1 in the aortic root in normal vessels of C57BL/ 6 mice $(\mathbf{a}-\mathbf{c})$ and atherosclerotic lesions of apoE $E^{-/-}$mice $(\mathbf{d}-\mathbf{f})$. $\mathrm{HA}$ deposition was localized using HA-BP to the endothelial layer of the nondiseased vessel of C57BL/ 6 mice (a), whereas HA was expressed on endothelium and throughout the atherosclerotic lesion of apoE-deficient mice (d). Serial sections immunostained for VCAM-1 (b and $\mathbf{e}$ ) demonstrate that the increased expression of VCAM-1 on SMCs within atherosclerotic lesions of $a p o E^{-/-}$mice (e) colocalizes with the deposition of $\mathrm{HA}(\mathbf{d})$ and is absent in disease-free vessels of C57BL/ 6 mice (b). Predigestion with hyaluronidase (Hase) abolished reactivity with $\mathrm{HA-BP}(\mathbf{c}, \mathbf{f})$.

advanced complex lesions (Figure 5, a, b, d, and e) containing foam cell-rich areas as well as fibrotic areas (demarcated by staining with anti-laminin) in the neointima (Figure $5 \mathrm{~g}$ ) or early lesions consisting mainly of foam cells (Figure 5, c and f). Thus, CD44 is required for maximal upregulation of VCAM-1 expression on vascular SMCs within atherosclerotic lesions. Note that VCAM-1 (Figures 3 and 5) as well as ICAM-1 and PECAM-1 (data not shown) were expressed at comparable levels on endothelial cells (arrowheads) in both CD44 wild-type, heterozygote, and CD44-null mice.

Since HA is the principal ligand of CD44, we investigated whether HA regulates the phenotype and acti- vation state of primary aortic SMC in vitro. At sites of inflammation, an immunostimulatory form of HA accumulates that is characterized by its lowmolecular-weight (LMW-HA) compared with the constitutively expressed highmolecular-weight form of HA (HMW-HA) (27). We found that VCAM-1 expression was upregulated approximately eightfold by treatment with LMW-HA, but not HMW-HA (Figure 6a). The effect was both dose- and time-dependent (Figure 6, b and c). HA-induced VCAM-1 expression was also dependent on nascent RNA and protein expression as it was inhibited by actinomycin $\mathrm{D}$ and cycloheximide (data not shown). The VCAM-1 promoter is strongly dependent upon NF- $\kappa B$ (28), and electromobility shift analysis indicated that LMW-HA induced a shift of an oligonucleotide containing the consensus sequence of the NF- $\mathrm{kB}$-binding site (Figure $6 \mathrm{~d}$ ) consistent with activation of NF-kB-binding activity. These data implicate HA binding to CD44 in the induction of VCAM-1 within atherosclerotic lesions.

$H A$ regulates the proliferation of primary aortic SMCs. A hallmark of the synthetic phenotype of SMCs is proliferation. Since the extracellular milieu controls the G1 phase of the cell cycle, we synchronized primary aortic SMCs into G0 by serum starvation and determined if HA affected their progression through the G1 phase. We found that LMW-HA induced a dosedependent proliferation of SMCs. LMW-HA was strongly mitogenic, inducing proliferation of SMCs almost as effectively as serum. The effects of LMW-HA and serum were not additive (Figure $7 \mathrm{a}$ ). In contrast,
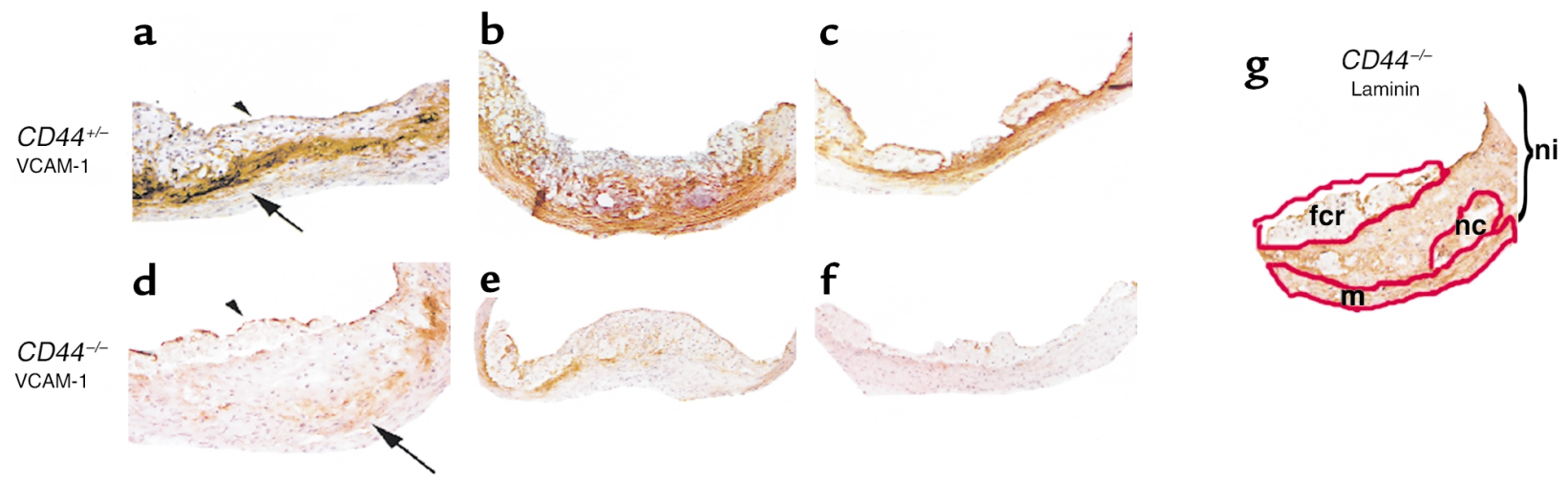

\section{Figure 5}

Induction of SMC VCAM-1 expression in atherosclerotic lesions is CD44-dependent. Expression of VCAM-1 in intermediate to advanced (a, $\mathbf{b}$, $\mathbf{d}, \mathbf{e})$ and early foam cell $(\mathbf{c}, \mathbf{f})$ lesions in the aortic root of $C D 44^{+/-}$apo $E^{-/-}(\mathbf{a}-\mathbf{c})$ and $C D 44^{-/-}$apo $E^{-/-}$mice $(\mathbf{d}-\mathbf{f})$ was compared. VCAM-1 expression was detected on endothelial cells (arrowhead) and SMCs (arrow) in $C D 44^{+/-}$apoE $E^{-/-}$mice (a) and on endothelial cells (arrowhead) but not

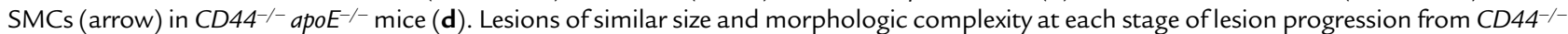
and $C D 44^{+/-}$mice are compared side by side. The architecture of an intermediate/advanced lesion from a CD44-/- mouse is detailed (g) in which a serial section of the section depicted in $\mathbf{d}$ was immunostained with an anti-laminin Ab. The large neointima (ni) contains a macrophage-rich foam cell region ( $\mathrm{fcr}$ ) layered above a matrix-rich layer containing a necrotic core $(\mathrm{nc})$. The media is intact, but diminished in size ( $\mathrm{m})$. All panels $\times 200$. Results are representative of the seven CD44-null and nine heterozygote mice analyzed. 
a

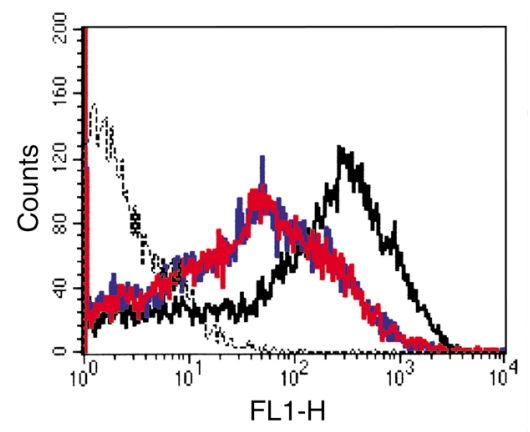

b

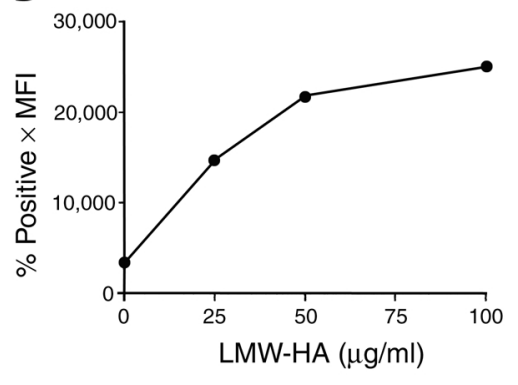

d

C

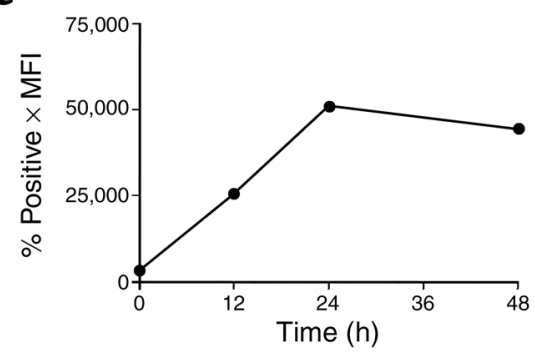

\section{Figure 6}

LMW-HA induces activation of cultured murine aortic SMCs. Vascular SMCs from C57BL/ 6 mice were left untreated (UT) or treated with LMW-HA or HMW-HA and then analyzed for expression of VCAM-1 and proliferation. (a) Flow cytometric analysis of VCAM- 1 expression of untreated cells (blue line), or cells treated with $100 \mu \mathrm{g} / \mathrm{ml}$ of LMW-HA (black) or HMW-HA (red) for 18 hours. Dashed line represents level of background fluorescence with an isotype-matched control $\mathrm{Ab}$. LMW-HA induced VCAM-1 expression is dose(b) and time-dependent (c) (data expressed as percentage of positive cells $\times$ mean fluorescent intensity $[\mathrm{MFI}])$. Treatment of SMCs with either LMW-HA orTNF for 2 hours induces a shift of an oligonucleotide of the consensus NF-KB-binding sequence (d). Data shown are representative of between three and five experiments. not only did HMW-HA not induce proliferation of serum-deprived SMCs, but it markedly inhibited serum-stimulated proliferation (Figure 7b). This modulation of proliferation by HA was not abrogated by pretreatment of the HA with proteinase $\mathrm{K}$, indicating the regulation was not due to contaminating proteins. Together these data suggest that LMW-HA is a potent stimulator of SMC proliferation whereas HMW-HA may play a role in maintaining SMCs in a quiescent state. The mitotic response to LMW-HA was dramatically reduced in SMCs derived from CD44deficient mice (65\%) and was also inhibited by antiCD44 Ab's (53\%) while isotype-matched irrelevant Ab's had no effect on LMW-HA-induced proliferation (Figure 7c). Similarly, the inhibition of seruminduced proliferation by HMW-HA was also reduced in SMCs derived from CD44-deficient mice (50\% inhibition in wild-type mice vs. $25 \%$ in CD44-null SMCs). Anti-CD44 Ab's reversed the inhibition of proliferation by HMW-HA in the case of wild-type mice (Figure $7 \mathrm{~d}$ ), but did not effect the response in SMCs derived from CD44-deficient mice. Together these data indicate that HA may be an important regulator of SMCs within both normal and atherosclerotic vessels through its interaction with CD44.

Macrophage recruitment to atherosclerotic lesions is CD44dependent. CD44 can mediate the initial rolling of activated T lymphocytes on endothelium under physiologic shear stress through binding to activated endothelial cell-associated HA (5). Therefore, we hypothesized that CD44 may also play a critical role in the migration of monocytes to atherosclerotic lesions. Since sufficient numbers of peripheral blood monocytes cannot be isolated from murine blood, thiogly- collate-elicited peritoneal macrophages are often used as a surrogate for determining the mechanisms that mediate monocyte cell recruitment to atherosclerotic lesions (22). We therefore compared the migration of CD44 wild-type and CD44-null peritoneal macrophages to atherosclerotic lesions. At 2 hours after adoptive transfer into apoE-deficient mice, comparable numbers of radiolabeled CD44-null and wild-type macrophages were detected in the spleens and livers of recipient animals. However, migration of CD44-null macrophages into the aortic arch was reduced by $60 \%$ compared with migration of CD44 wild-type macrophages $(P<0.05, n=5 /$ group; Figure $8 a)$. Since the macrophage is a further differentiated cell than the peripheral blood monocyte, we also compared the ability of undifferentiated hematopoietic cells (including monocytes) isolated from the bone marrow of CD44 wild-type and CD44-deficient mice to migrate to atherosclerotic lesions. Migration of CD44-null hematopoietic cells into the aortic arch was reduced $48 \%$ compared with the migration of CD44 wild-type cells (Figure 8b; $P<0.05, n=9$ ). To determine whether CD44 may also affect either the survival or retention of macrophages within lesions we analyzed a second group of mice 48 hours after transfer of fluorescently labeled macrophages. At this later time point, 90\% fewer CD44-null macrophages were detected in the lesions of the arch compared with CD44 wild-type macrophages $(P<0.05, n=7 /$ group; Figure $8 c)$. These data establish that CD44 plays a critical role in the recruitment of macrophages to atherosclerotic lesions and suggest that CD44 may play an additional role in the survival or retention of inflammatory cells within atherosclerotic lesions. 

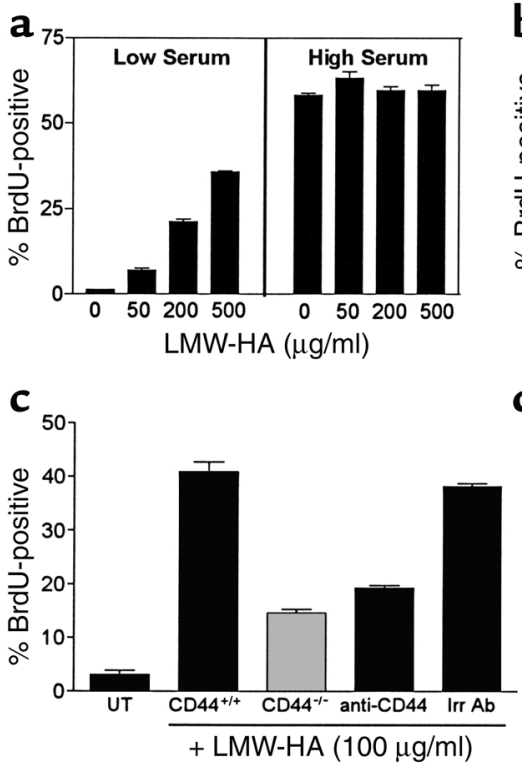

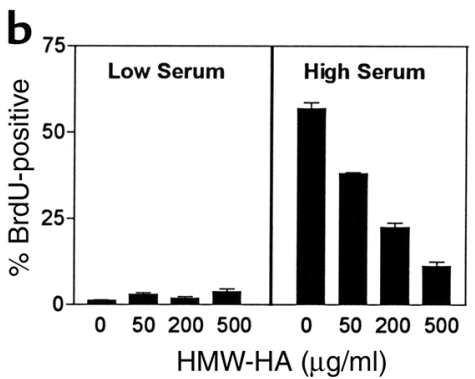

d

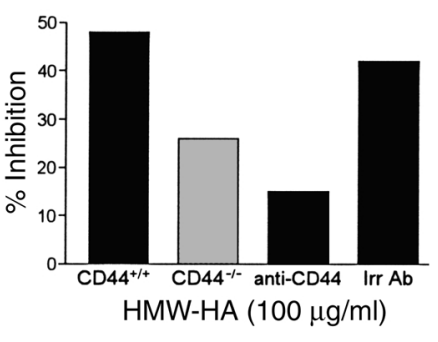

\section{Figure 7}

LMW-HA and HMW-HA differentially regulate SMC proliferation. SMCs were treated with increasing doses of either LMW-HA or HMW-HA in the presence of low (1\%) or high (10\%) serum. (a) LMW-HA induced dosedependent proliferation of SMCs cultured in low serum but did not further enhance proliferation induced by high serum. (b) HMW-HA failed to induce proliferation of SMCs cultured in low serum but inhibited serum-induced proliferation in a dose-dependent manner. The induction of proliferation by LMW-HA (c) as well as the inhibition of serum-induced proliferation by HMW-HA (d) is mediated to a substantial degree by CD44 because the induction of proliferation by LMW-HA is reduced in CD44-deficient SMCs (gray bars) as well as CD44 wild-type SMCs (black bars) cultured in the presence of anti-CD44 Ab (anti-CD44), but not an isotype-matched irrelevant $A b$ (Irr $A b)$.

\section{Discussion}

We demonstrate that genetic deficiency in CD44 results in marked reduction in atherosclerosis in apoE-deficient mice. The reduction in atherosclerotic lesions in CD44-null mice represents the greatest reduction in lesion size resulting from mutation of any single gene reported to date (29). Furthermore, our data indicate that CD44 promotes atherogenesis through multiple mechanisms, including recruitment of macrophages to atherosclerotic lesions and activation of vascular SMCs. Several previous studies indicated that CD44 promotes a variety of chronic inflammatory responses. Specifically, administration of anti-CD44 Ab's to mice reduced the inflammation associated with delayed-type hypersensitivity (23), arthritis (30-32), and experimental allergic encephalomyelitis (33). The recent development of CD44-mutant mice allows a genetic approach to determining the role of CD44 in inflammatory diseases. Mice deficient in CD44 exon 7 have reduced inflammation associated with experimental colitis (34). To date only the delayed-type hypersensitivity model has been studied in the CD44-null mouse with no difference detected between the wild-type and CD44-deficient mice (17). The discrepancy between the results from the Ab study and the knockout mouse indicate that either compensatory mechanisms or redundant pathways are present in the CD44-null mouse that are sufficient to mount a delayed-type hypersensitivity response. However, we now show that atherosclerosis is markedly reduced in CD44-null mice, providing genetic evidence that CD44 is critical to the vascular inflammation associated with atherosclerosis.

The mechanism by which Ab's against CD44 inhibit chronic inflammation are not fully defined but potentially involve leukocyte recruitment and leukocyte and parenchymal cell activation. CD44 mediates the rolling of activated $\mathrm{T}$ lymphocytes on activated endothelial cells through an HA-dependent mecha- a

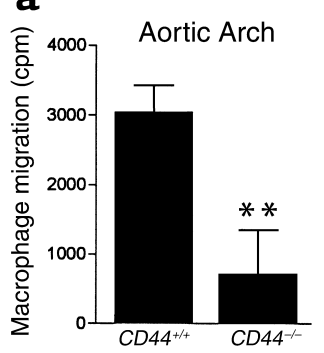

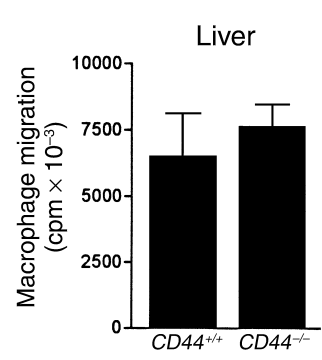

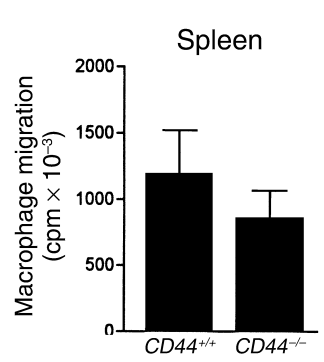

b

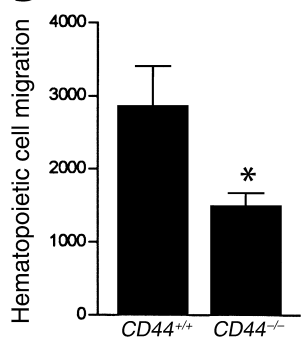

C

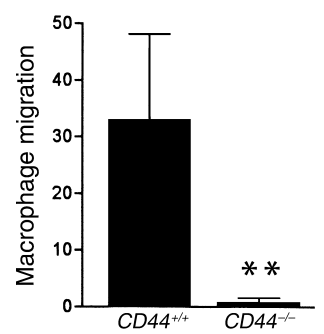

Figure 8

Macrophage recruitment to atherosclerotic lesions is CD44-dependent. Either thioglycollate-elicited peritoneal macrophages (a and $\mathbf{c}$ ) or bone marrow-derived hematopoietic cells (b) were labeled and injected into 24- to 28-week-old apoE $E^{-/-} \mathrm{CD} 44^{+/+}$recipient mice. (a) Two hours after transfer, $60 \%$ fewer ${ }^{111}$ indium-labeled $C D 44^{-/-}$macrophages migrated to the aortic arch compared with the migration of $C D 44^{+/+}$macrophages, while comparable numbers of $C D 44^{-/-}$and $C D 44^{+/+}$macrophages were detected in the spleens and livers of recipient mice. (b) Two hours after transfer, $48 \%$ fewer calcein-labeled $C D 44^{-/-}$hematopoietic cells were detected in the aortic arch of recipient mice relative to the migration of $\mathrm{CD} 44^{+/+}$cells. (c) Forty-eight hours after transfer, 90\% fewer $C D 44^{-/-}$macrophages were detected in atherosclerotic lesions of $a p o E^{-/-}$compared with $C D 44^{+/+}$macrophages. ${ }^{*} P<0.05 ;{ }^{*} P<0.01$. 
nism $(5,6)$. This rolling event is a requisite step for migration of leukocytes to sites of inflammation. While the role of specific adhesion molecules in granulocyte and lymphocyte trafficking have been demonstrated through the use of blocking Ab's, there have been few studies examining the contribution of individual adhesion proteins to monocyte/macrophage recruitment. In this study we found that in addition to T lymphocytes, CD44 promotes the recruitment of inflammatory cells to sites of chronic inflammation. This is consistent with the reduction in total lesion size observed at both 20 and 24 weeks that correlates with fewer total number of macrophages in these lesions. In similar studies, Ab's to ICAM-1 also inhibited macrophage accumulation within atherosclerotic lesions of $a p o E^{-/-}$mice by $65 \%$ (22). Furthermore, anti-P-selectin-specific and anti-VCAM-1-specific $A b$ 's reduced firm adhesion of macrophages to aorta of apoE $E^{-/-}$mice under shear stress (35). Similar experiments using aorta from hypercholesterolemic Watanabe rabbits demonstrated that CD43, leukosialin, may also mediate macrophage adhesion (36). A role for other adhesion molecules in atherosclerosis has also been demonstrated using gene-deficient strains including ICAM-1 $(37,38)$ and E- and P-selectin $(37$, 39). It is known that multiple adhesion/ligand pairs contribute to the recruitment of leukocytes, and therefore it is not surprising that no one pair of adhesion receptors is responsible for recruitment of monocytes/macrophages to atherosclerotic lesions. These studies identify CD44 as an additional adhesion receptor that plays a critical role in the accumulation of macrophages in the vessel wall. Importantly, CD44 is not required for migration of lymphocytes (23) or macrophages (this study) to secondary lymphoid organs demonstrating a selective role for CD44 in leukocyte trafficking to chronic sites of inflammation. We therefore suggest that targeting of CD44/HA interactions may provide a strategy for preventing progression of atherosclerosis and other chronic inflammatory diseases without disrupting homeostatic recirculation of immune cells.

CD44 and HA have also been shown to regulate parenchymal cell function. A characteristic feature of atherosclerotic lesions is transition of SMCs from a contractile to a synthetic phenotype. The synthetic phenotype is characterized by changes in gene expression (increased adhesion molecule and decreased $\alpha$-smooth muscle actin) (4), as well as proliferation and migration of medial SMCs into the neointima $(3,26,40)$. Importantly, CD44 was shown to promote human SMC proliferation (14). Furthermore, it was recently reported that expression of high levels of CD44 on cultured murine vascular SMCs is maintained by the production of endogenous endothelin and that an endothelin-converting enzyme inhibitor blocked HA-induced vascular SMC proliferation (41), further supporting CD44/HA interactions in SMC activation. In this study we demonstrated that LMW-HA also induced increased expression of VCAM- 1 on SMCs in vitro and that CD44 is required for the maximal upregulation of VCAM-1 on SMCs in murine atherosclerotic lesions. HA-mediated induction of VCAM-1 expression on SMCs may be induced, at least in part, through the activation of $\mathrm{NF}-\kappa \mathrm{B}$, which also mediates activation of macrophages following ligation of CD44 (42).

Similar to the findings in human SMCs (14), we now report that LMW-HA induces the proliferation of murine SMCs through a CD44-dependent mechanism. Of particular interest is our finding that the ability of HA to stimulate SMC activation is dependent upon the form of HA. Thus, the LMW-HA that accumulates during an inflammatory response through either degradation of HMW-HA by hyaluronidase or oxygen/nitrogen radicals (43) or through de novo synthesis by HA synthase (44), activated SMC VCAM-1 expression and proliferation, while HMW-HA had no effect on VCAM-1 expression and was a potent inhibitor of serum-induced proliferation. Taken together, these data suggest that HMWHA may contribute to maintenance of the contractile state of SMCs while LMW-HA may promote dedifferentiation to the synthetic state. However, although HA is upregulated within atherosclerotic lesions, it is unclear whether all or part of the proatherogenic effects of CD44, which has affinity for additional ligands, including osteopontin (45) and fibronectin (46), are mediated through interaction with HA. Similarly, while we have demonstrated that the effects of HA on SMC differentiation and activation are mediated to a substantial degree by CD44-dependent mechanisms, other HA-binding proteins, including Rhamm (47)and TSG-6 (48), may also contribute to these processes. It will be of particular interest to characterize the form of HA that is present in normal versus atherosclerotic vessels in view of the distinct biologic activities of LMW-HA and HMW-HA.

In this report, we demonstrate a profound impact of CD44 deficiency on atherosclerotic lesion formation. Two mechanisms by which CD44 promotes atherogenesis are the recruitment of macrophages to atherosclerotic lesions and regulation of the phenotypic dedifferentiation of vascular SMCs. However, it is important to note that CD44 may promote atherogenesis through additional mechanisms, including the activation of macrophages. Thus, ligation of CD44 by HA on macrophages is known to induce production of soluble inflammatory mediators including IL-12 (8), MCP-1 (9), and iNOS (10), all of which have been implicated in the pathogenesis of atherosclerosis (49-54). Furthermore, since CD44 is known to mediate the rolling of $\mathrm{T}$ lymphocytes on endothelium, CD44 may also mediate recruitment of $T$ lymphocytes to lesions where they are known to play a role during atherogenesis (55-57). Furthermore, although CD44 can directly activate SMCs, CD44-dependent activation of SMCs may also be mediated in part through indirect pathways. We showed that CD44 mediates 
$\mathrm{T}$ cell adherence to airway SMCs that induce DNA synthesis (7). Furthermore, CD44 acts as a costimulatory molecule on T lymphocytes (58). Therefore, some HA-induced activation events in vivo may be due to $\mathrm{T}$ cell-mediated signals. In atherosclerotic lesions, both $\mathrm{T}$ lymphocytes and macrophages are in contact with SMCs, and it will be important to determine the contribution of these potential interactions toward regulation of SMC function.

In conclusion, these studies suggest that inhibitors of CD44 function and CD44/HA (59) interactions may provide an effective means for inhibiting chronic inflammatory diseases, including atherosclerosis.

\section{Acknowledgments}

This work was supported by Public Health Service grants HL57811, AI34412, AI458113, HL62250, HL66250 (R. Assoian), and CA09140 (C. Cuff), and American Heart Association grant 60182U (C. Cuff) and fellowship $20160 \mathrm{U}$ (D. Kothapalli). The authors wish to thank Tak Mak for providing the CD44-null mice and Erin Moss, Craig Farrel, Naisha Miller, and Irene Crichton for technical assistance.

1. Ross, S.D., et al. 1999. Clinical outcomes in statin treatment trials: a meta-analysis. Arch. Intern. Med. 159:1793-1802.

2. Ross, R. 1999. Atherosclerosis: an inflammatory disease. N. Engl. J. Med. 340:115-126.

3. Braun, M., Pietsch, P., Schror, K., Baumann, G., and Felix, S.B. 1999. Cellular adhesion molecules on vascular smooth muscle cells. Cardiovasc. Res. 41:395-401.

4. Rolfe, B.E., Muddiman, J.D., Smith, N.J., Campbell, G.R., and Campbell, J.H. 2000. ICAM-1 expression by vascular smooth muscle is phenotypedependent. Atherosclerosis. 149:99-110.

5. DeGrendele, H.C., Estess, P., Picker, L.J., and Siegelman, M.H. 1996. CD44 and its ligand hyaluronate mediate rolling under physiologic flow: a novel lymphocyte-endothelial cell primary adhesion pathway. J. Exp. Med. 183:1119-1130.

6. DeGrendele, H.C., Estess, P., and Siegelman, M.H. 1997. Requirement for CD44 in activated T cell extravasation into an inflammatory site. Science. 278:672-675.

7. Lazaar, A.L., et al. 1994. T lymphocytes adhere to airway smooth muscle cells via integrins and CD44 and induce smooth muscle cell DNA synthesis. J. Exp. Med. 180:807-816.

8. Hodge-Dufour, J., et al. 1997. Induction of IL-12 and chemokines by hyaluronan requires adhesion dependent priming of resident but not elicited macrophages. J. Immunol. 159:2492-2500.

9. McKee, C.M., Penno, M.B., Cowman, M., Bao, C., and Noble, P.W. 1996. Hyaluronan (HA) fragments induce chemokine gene expression in alveolar macrophages. The role of HA size and CD44. J. Clin. Invest. 98:2403-2413.

10. McKee, C.M., et al. 1997. Hyaluronan fragments induce nitric oxide synthase in murine macrophages through a nuclear factor $\kappa \mathrm{B}$-dependent mechanism. J. Biol. Chem. 272:8013-8018.

11. Galandrini, R., et al. 1993. Antibodies to CD44 trigger effector functions of human T cell clones. J. Immunol. 150:4225-4235.

12. Guo, Y., et al. 1996. Identification of a costimulatory molecule rapidly induced by CD40L as CD44H. J. Exp. Med. 184:955-961.

13. Huet, S., et al. 1989. CD44 contributes to T cell activation. J. Immunol. 143:798-801.

14. Jain, M., et al. 1996. Role of CD44 in the reaction of vascular smooth muscle cells to arterial wall injury. J. Clin. Invest. 97:596-603.

15. Evanko, S.P., Raines, E.W., Ross, R., Gold, L.I., and Wight, T.N. 1998. Proteoglycan distribution in lesions of atherosclerosis depends on lesion severity, structural characteristics, and the proximity of platelet-derived growth factor and transforming growth factor- $\beta$. Am. J. Pathol. 152:533-546.

16. Riessen, R., Wight, T.N., Pastore, C., Henley, C., and Isner, J.M. 1996. Distribution of hyaluronan during extracellular matrix remodeling in human restenotic arteries and balloon-injured rat carotid arteries. Circulation. 93:1141-1147.

17. Schmits, R., et al. 1997. CD44 regulates hematopoietic progenitor distribution, granuloma formation and tumorigenicity. Blood. 90:2217-2233.
18. Tsukamoto, K., Tangirala, R., Chun, S., Puré, E., and Rader, D.J. 1999. Rapid regression of atherosclerosis induced by liver-directed gene transfer of ApoE in ApoE-deficient mice. Arterioscler. Thromb. Vasc. Biol. 19:2162-2170.

19. Gerdes, L.U., Gerdes, C., Klausen, I.C., and Faergeman, O. 1992. Generation of analytic plasma lipoprotein profiles using two pre-packed Superose 6B columns. Clin. Chim. Acta. 205:1-9.

20. Amrani, Y., Lazaar, A.L., and Panettieri, R.A. 1999. Up-regulation of ICAM-1 by cytokines in human tracheal smooth muscle cells involves an $\mathrm{NF}-\kappa \mathrm{B}-$ dependent signaling pathway that is only partially sensitive to dexamethasone. J. Immunol. 163:2128-2134.

21. Roovers, K., Davey, G., Zhu, X., Bottazzi, M.E., and Assoian, R.K. 1999. $\alpha 5 \beta 1$ integrin controls cyclin D1 expression by sustaining MAP kinase activity in growth factor-treated cells. Mol. Biol. Cell. 10:3197-3204.

22. Patel, S.S., Thiagarajan, R., Willerson, J.T., and Yeh, E.T.H. 1998. Inhibition of $\alpha_{4}$ integrin and ICAM-1 markedly attenuate macrophage homing to atherosclerotic plaques in ApoE-deficient mice. Circulation. 97:75-81.

23. Camp, R.L., Scheynius, A., Johansson, C., and Puré, E. 1993. CD44 is necessary for optimal contact allergic responses but is not required for normal leukocyte extravasation. J. Exp. Med. 178:497-508.

24. Underhill, C.B., and Zhang, L. 2000. Analysis of hyaluronan using biotinylated hyaluronan-binding proteins. Methods Mol. Biol. 137:441-447.

25. van der Wal, A.C., Das, P.K., Tiggers, A.J., and Becker, A.E. 1992. Adhesion molecules on the endothelium and mononuclear cells in human atherosclerotic lesions. Am. J. Pathol. 141:1427-1433.

26. Iiyama, K., et al. 1999. Patterns of vascular cell adhesion molecule-1 and intercellular adhesion molecule-1 expression in rabbit and mouse atherosclerotic lesions and at sites predisposed to lesion formation. Circ. Res. 85:199-207.

27. Fraser, J.R., Laurent, T.C., and Laurent, U.B. 1997. Hyaluronan: its nature, distribution, functions and turnover. J. Intern. Med. 242:27-33.

28. Neish, A.S., Williams, A.J., Palmer, H.J., Whitley, M.Z., and Collins, T. 1992. Functional analysis of the human vascular cell adhesion molecule 1 promoter. J. Exp. Med. 176:1583-1593.

29. Knowles, J.W., and Maeda, N. 2000. Genetic modifiers of atherosclerosis in mice. Arterioscler. Thromb. Vasc. Biol. 20:2236-2345.

30. Mikecz, K., Brennan, F.R., Kim, J.H., and Glant, T.T. 1995. Anti-CD44 treatment abrogates tissue oedema and leukocyte infiltration in murine arthritis. Nat. Med. 1:558-563.

31. Nedvetzki, S., et al. 1999. CD44 involvement in experimental collageninduced arthritis (CIA). J. Autoimmun. 13:39-47.

32. Mikecz, K., Dennis, K., Shi, M., and Kim, J.H. 1999. Modulation of hyaluronan receptor (CD44) function in vivo in a murine model of rheumatoid arthritis. Arthritis Rheum. 42:659-668.

33. Brocke, S., Piercy, C., Steinman, L., Weissman, I.L., and Veromaa, T. 1999. Antibodies to CD44 and integrin alpha4, but not L-selectin, prevent central nervous system inflammation and experimental encephalomyelitis by blocking secondary leukocyte recruitment. Proc. Natl. Acad. Sci. USA. 96:6896-6901.

34. Wittig, B.M., Johansson, B., Zoller, M., Schwarzler, C., and Gunthert, U. 2000. Abrogation of experimental colitis correlates with increased apoptosis in mice deficient for CD44 variant exon 7 (CD44v7). J. Exp. Med. 191:2053-2064.

35. Ramos, C.L., et al. 1999. Direct demonstration of P-selectin- and VCAM1 -dependent mononuclear cell rolling in early atherosclerotic lesions in apolipoprotein E-deficient mice. Circ. Res. 84:1237-1244.

36. McEvoy, L.M., Jutila, M.A., Tsao, P.S., Cooke, J.P., and Butcher, E.C. 1997. Anti-CD43 inhibits monocyte-endothelial adhesion in inflammation and atherogenesis. Blood. 90:3587-3594.

37. Beaudet, A.L., Velji, R., Guevara, N.V., Hicks, M.J., and Chan, L. 2000. PSelectin or intercellular adhesion molecule (ICAM)-1 deficiency substantially protects against atherosclerosis in apolipoprotein E-deficient mice. J. Exp. Med. 191:189-194.

38. Nageh, M.F., et al. 1997. Deficiency of inflammatory cell adhesion molecules protects against atherosclerosis in mice. Arterioscler. Thromb. Vasc. Biol. 17:1517-1520.

39. Dong, Z.-M., Brown, A.A., and Wagner, D.D. 2000. Prominent role of Pselectin in the development of advanced atherosclerosis in ApoE-deficient mice. Circulation. 101:2290-2295.

40. Li, H., Cybulsky, M.I., Gimbrone, M.A., and Libby, P. 1993. Inducible expression of vascular cell adhesion molecule- 1 by vascular smooth muscle cells in vitro and within rabbit atheroma. Am. J. Pathol. 143:1551-1559.

41. Tanaka, Y., Makiyama, Y., and Mitsui, Y. 2000. Endothelin-1 is involved in the growth promotion of vascular smooth cells by hyaluronic acid. Int. J. Cardiol. 76:39-47.

42. Noble, P.W., McKee, C.M., Cowman, M., and Shin, H.S. 1996. Hyaluronan fragments activate an NF- $\mathrm{BB} / \mathrm{I}-\kappa \mathrm{B} \alpha$ autoregulatory loop in murine macrophages. J. Exp. Med. 183:2373-2378.

43. Li, M., Rosenfeld, L., Vilar, R.E., and Cowman, M.K. 1997. Degradation 
of hyaluronan by peroxynitrite. Arch. Biochem. Biophys. 341:245-250.

44. Papakonstantinou, E., Karakiulakis, G., Roth, M., and Block, L.H. 1995 Platelet-derived growth factor stimulates the secretion of hyaluronic acid by proliferating human vascular smooth muscle cells. Proc. Natl. Acad. Sci. USA. 92:9881-9885.

45. Weber, G.F., Ashkar, S., Glimcher, M.J., and Cantor, H. 1996. Receptorligand interaction between CD44 and osteopontin (Eta-1). Science. 271:509-512.

46. Jalkanen, S., and Jalkanen, M. 1992. Lymphocyte CD44 binds the $\mathrm{COOH}$-terminal heparin-binding domain of fibronectin. J. Cell Biol. 116:817-825.

47. Savani, R.C., et al. 1995. Migration of bovine aortic smooth muscle cells after wounding injury. The role of hyaluronan and RHAMM. J. Clin. Invest. 95:1158-1168.

48. Lee, T.H., Wisniewski, H.G., and Vilcek, J. 1992. A novel secretory tumor necrosis factor-inducible protein (TSG-6) is a member of the family of hyaluronate binding proteins, closely related to the adhesion receptor CD44. J. Cell Biol. 116:545-557.

49. Lee, T.-S., Yen, H.-C., Pan, C.-C., and Chau, L.-Y. 1999. The role of interleukin 12 in the development of atherosclerosis in ApoE-deficient mice. Arterioscler. Thromb. Vasc. Biol. 19:734-742.

50. Gu, L., et al. 1998. Absence of monocyte chemoattractant protein-1 reduces atherosclerosis in low density lipoprotein receptor-deficient mice. Mol. Cell. 2:275-281.

51. Gosling, J., et al. 1999. MCP-1 deficiency reduces susceptibility to atherosclerosis in mice that overexpress human apolipoprotein B.
J. Clin. Invest. 103:773-778

52. Dawson, T.C., Kuziel, W.A., Osahar, T.A., and Maeda, N. 1999. Absence of CC chemokine receptor-2 reduces atherosclerosis in apolipoprotein E-deficient mice. Atherosclerosis. 143:205-211.

53. Boring, L., Gosling, J., Cleary, M., and Charo, I.F. 1998. Decreased lesion formation in CCR2-/- mice reveals a role for chemokines in the initiation of atherosclerosis. Nature. 394:894-897.

54. Detmers, P.A., et al. 2000. Deficiency in inducible nitric oxide synthase results in reduced atherosclerosis in apolipoprotein E-deficient mice. $J$. Immunol. 165:3430-3435.

55. Daugherty, A., et al. 1997. The effects of total lymphocyte deficiency on the extent of atherosclerosis in apolipoprotein E-/- mice. J. Clin. Invest. 100:1575-1580.

56. Dansky, H.M., Charlton, S.A., Harper, M.M., and Smith, J.D. 1997. T and B lymphocytes play a minor role in atherosclerotic plaque formation in the apolipoprotein E-deficient mouse. Proc. Natl. Acad. Sci. USA. 94:4642-4646.

57. Zhou, X., Nicoletti, A., Elhage, R., and Hansson, G.K. 2000. Transfer of CD4(+) $\mathrm{T}$ cells aggravates atherosclerosis in immunodeficient apolipoprotein E knockout mice. Circulation. 102:2919-2922.

58. Galandrini, R., Galluzzo, E., Albi, N., Grossi, C.E., and Velardi, A. 1994. Hyaluronate is costimulatory for human $\mathrm{T}$ cell effector functions and binds to CD44 on activated T cells. J. Immunol. 153:21-31.

59. Mummert, M.E., Mohamadzadeh, M., Mummert, D.I., Norikatsu, M., and Takashima, A. 2000. Development of a peptide inhibitor of hyaluronan-mediated leukocyte trafficking. J. Exp. Med. 192:769-780. 